voL. 32 (1985), 161-176.

\title{
MEROMORPHIC UNIVALENT FUNCTIONS WITH POSITIVE COEFFICIENTS
}

\author{
M.L. Mogra, T.R. Reddy and 0.P. Juneja
}

\begin{abstract}
For the class of meromorphically starlike functions of prescribed order, the concept of type has been introduced. A characterization of meromorphically starlike functions of order $\alpha$ and type $\beta$ has been obtained when the coefficients in its Laurent series expansion about the origin are all positive. This leads to a study of coefficient estimates, distortion theorems, radius of convexity estimates, integral operators, convolution properties et cetera for this class. It is seen that the class considered demonstrates, in some respects, properties analogous to those possessed by the corresponding class of univalent analytic functions with negative coefficients.
\end{abstract}

\section{Introduction}

Let $S$ denote the class of functions of the form

$$
g(z)=z+\sum_{n=2}^{\infty} b_{n} z^{n}
$$

that are analytic and univalent in $U=\{z:|z|<1\}$. Denote by $S^{*}(\alpha)$ and $K(\alpha),(0 \leq \alpha<1)$ the subclasses of functions in $S$ that are starlike of order $\alpha$ and convex of order $\alpha$ respectively. Analytically, $g \in S^{\star}(\alpha)$,

Received 7 January 1985.

Copyright Clearance Centre, Inc. Serial-fee code: 0004-9727/85 $\$ A 2.00+0.00$. 
if and only if, $g$ is of the form (l.l) and satisfies

$$
\operatorname{Re}\left\{\frac{z g^{\prime}(z)}{g(z)}\right\}>\alpha, z \in U
$$

similarly, $g \in K(\alpha)$, if and only if, $g$ is of the form (1.1) and satisfies

$$
\operatorname{Re}\left\{1+\frac{z g^{\prime \prime}(z)}{g^{\prime}(z)}\right\}>\alpha
$$

Let $T$ denote the class of functions analytic in $U$ that are of the form

$$
g(z)=z-\sum_{n=2}^{\infty} b_{n} z^{n}, b_{n} \geq 0 .
$$

and let $T^{*}(\alpha)=T \cap S^{*}(\alpha), C(\alpha)=T \cap K(\alpha)$. The class $T^{*}(\alpha)$ and allied classes possess some very interesting properties and have been recently studied by Silverman $([15],[16])$, Silverman and Silvia ([17]) and others. Gupta and Jain [4] extended some of the results of Silverman to functions of the form (1.4) that are starlike of order $\alpha$ and type $\beta$ $(0<\beta \leq 1)$. The class of starlike functions of order $\alpha$ and type $\beta$ was introduced by Juneja and Mogra ([5]) who also made a detailed study about it $([6],[9])$.

Let $\Sigma$ denote the class of functions of the form

$$
f(z)=\frac{1}{z}+\sum_{n=1}^{\infty} a_{n} z^{n}
$$

which are regular in $D=\{z: 0<|z|<1\}$ with a simple pole at the origin with residue 1 there. Let $\Sigma_{S}, \Sigma^{*}(\alpha)$ and $\Sigma_{K}(\alpha)(0 \leq \alpha<1)$ denote the subclasses of $\Sigma$ that are univalent, meromorphically starlike of order $\alpha$ and meromorphically convex of order a respectively. Analytically $f$, of the form $(1.5)$, is in $\Sigma^{\star}(\alpha)$, if and only if,

$$
\operatorname{Re}\left\{-\frac{z f^{\prime}(z)}{f(z)}\right\}>\alpha, z \in U \text { : }
$$

similarly, $f \in \Sigma_{K}(\alpha)$, if and only if, $f$ is of the form (1.5) and satisfies 


$$
\operatorname{Re}\left\{-\left(1+\frac{z f^{\prime \prime}(z)}{f^{\prime}(z)}\right)\right\}>\alpha, z \in U
$$

The class $\Sigma^{*}(\alpha)$ and similar other classes have been extensively studied by Pommerenke [11], Clunie [2], Miller [8], Royster [13] and others.

Since, to a certain extent, the work in the meromorphic univalent case has paralleled that of regular univalent case, it is natural to search for a subclass of $\Sigma_{S}$ that has properties analogous to those of $T^{\star}(\alpha)$. To this end, Juneja and Reddy [7] introduced the class $\Sigma_{P}$ of functions of the form

$$
f(z)=\frac{1}{z}+\sum_{n=1}^{\infty} a_{n} z^{n}, a_{n} \geq 0
$$

that are regular and univalent in $D$. They showed that the class $\Sigma_{P}^{\star}(\alpha)=\Sigma_{P} \cap \Sigma^{\star}(\alpha)$ possesses properties analogous to those of $T^{*}(\alpha)$ and also pointed out the subtle differences between the two classes.

The aim of the present paper is first to introduce the class of meromorphically starlike functions of order $\alpha$ and type $\beta$ which we denote by $\Sigma^{\star}(\alpha, \beta)$. We then consider the class $\Sigma_{P}^{\star}(\alpha, \beta)=\Sigma_{P} \cap \Sigma^{\star}(\alpha, \beta)$ and extend some of the results of Juneja and Reddy [7] to this class. Some of the properties of this class will be seen to be analogous to those in [4]. We also study some other aspects such as convolution properties et cetera whose analogues have not been obtained in [4].

\section{Coefficient estimates}

We begin with the definition of meromorphically starlike functions of order $\alpha$ and type $B$.

DEFINITION. A function $f \in \Sigma$ is said to be meromorphically starlike of order $\alpha$ and type $B$ if it satisfies the condition

$$
\left|z \frac{f^{\prime}(z)}{f(z)}+1\right|<\left|\beta \frac{z f^{\prime}(z)}{f(z)}+2 \alpha-1\right|
$$

for some $\alpha, \beta(0 \leq \alpha<1,0<\beta \leq 1)$ and for all $z \in U$.

The class of meromorphically starlike functions of order $\alpha$ and type 
$\beta$ we denote by $\sum \star(\alpha, \beta)$.

It is easy to check that $\Sigma *(\alpha, 1)$ is the class of meromorphically starlike functions of order $\alpha ; \Sigma^{\star}(0,1)$ gives the whole class of meromorphically starlike functions whereas $\Sigma^{\star}(0, \beta)$ yields the class studied by Padmanabhan [10].

The following theorem gives a sufficient condition for a function to be in $\Sigma *(\alpha, \beta)$.

THEOREM 1. Let $f(z)=\frac{1}{z}+\sum_{n=1}^{\infty} a_{n} z^{n}$ be regular in $D$. If

$$
\sum_{n=1}^{\infty}[(1+\beta) n+(2 \alpha-1) \beta+1]\left|a_{n}\right| \leq 2 \beta(1-\alpha),
$$

$0 \leq \alpha<1,0<\beta \leq 1$, then $f \in \Sigma^{\star}(\alpha, \beta)$.

Proof. It is easy to see that (2.1) implies $f(z) \neq 0$ in $D$. Suppose (2.1) holds for all admissible values of $\alpha$ and $\beta$. Consider the expression

$$
H\left(f, f^{\prime}\right)=\left|z f^{\prime}(z)+f(z)\right|-\beta\left|z f^{\prime}(z)+(2 \alpha-1) f(z)\right| .
$$

Replacing $f$ and $f^{\prime}$ by their series expansions, we have for $0<|z|=r<1$.

$$
H\left(f, f^{\prime}\right)=\left|\sum_{n=1}^{\infty}(n+1) a_{n} z^{n}\right|-\beta\left|2(\alpha-1) \frac{1}{z}+\sum_{n=1}^{\infty}(n+2 \alpha-1) a_{n} z^{n}\right|
$$

or

$$
\begin{aligned}
r H\left(f, f^{\prime}\right) & \leq \sum_{n=1}^{\infty}(n+1)\left|a_{n}\right| r^{n+1}-\beta\left\{2(1-\alpha)-\sum_{n=1}^{\infty}(n+2 \alpha-1)\left|a_{n}\right| r^{n+1}\right\} \\
& =\sum_{n=1}^{\infty}[(1+\beta) n+(2 \alpha-1) \beta+1]\left|a_{n}\right| r^{n+1}-2 \beta(1-\alpha) .
\end{aligned}
$$

Since the above inequality holds for all $r, 0<r<1$, letting $r \rightarrow 1$, we have

$$
\begin{aligned}
H\left(f, f^{\prime}\right) & \leq \sum_{n=1}^{\infty}[(1+\beta) n+(2 \alpha-1) \beta]\left|a_{n}\right|-2 \beta(1-\alpha) \\
& \leq 0
\end{aligned}
$$


by (2.1). Hence it follows that $\left|\frac{z f^{\prime}(z)}{f(z)}+1\right|<\beta\left|\frac{z f^{\prime}(z)}{f(z)}+2 \alpha-1\right|$, so that $f \in \Sigma^{*}(\alpha, \beta)$. Hence the theorem.

Let us write $\Sigma_{P}^{*}(\alpha, \beta)=\Sigma_{P} \cap \Sigma^{*}(\alpha, \beta)$ where $\Sigma_{P}$ is the class of functions of the form (1.8) that are regular and univalent in $D$. Our next theorem shows that condition (2.1) is both necessary and sufficient for an $f$ to be in $\Sigma_{P}^{\star}(\alpha, \beta)$.

THEOREM 2. Let $f(z)=\frac{1}{z}+\sum_{n=1}^{\infty} a_{n} z^{n}, a_{n} \geq 0$, be regular in $D$. Then $f \in \Sigma_{P}^{\star}(\alpha, \beta)$ if and only if (2.1) is satisfied.

Proof. In view of Theorem 1 it is sufficient to show the 'only if' part. Let us assume that $f(z)=\frac{1}{z}+\sum_{n=1}^{\infty} a_{n} z^{n}, a_{n} \geq 0$, is in $\sum_{P}^{\star}(\alpha, \beta)$. Then

$$
\left|\frac{\frac{z f^{\prime}(z)}{f(z)}+1}{\frac{z f^{\prime}(z)}{f(z)}+2 \alpha-1}\right|=\left|\frac{\sum_{n=1}^{\infty}(n+1) a_{n} z^{n}}{2(1-\alpha) \frac{1}{z}-\sum_{n=1}^{\infty}(n+2 \alpha-1) a_{n} z n}\right|<\beta
$$

for all $z \in D$. Using the fact that $\operatorname{Re}(z) \leq|z|$ for all $z$, it follows that

$$
\operatorname{Re}\left\{\frac{\sum_{n=1}^{\infty}(n+1) a_{n}{ }^{2}}{2(1-\alpha) \frac{1}{z}-\sum_{n=1}^{\infty}(n+2 \alpha-1)}\right\}<B, z \in U .
$$

Now choose the values of $z$ on the real axis so that $\frac{z f^{\prime}(z)}{f(z)}$ is real. Upon clearing the denominator in (2.3) and letting $z \rightarrow 1$ through positive values, we obtain

$$
\sum_{n=1}^{\infty}(n+1) a_{n} \leq \beta\left(2(1-\alpha)-\sum_{n=1}^{\infty}(n+2 \alpha-1) a_{n}\right)
$$

or 


$$
\sum_{n=1}^{\infty}[(1+\beta) n+(2 \alpha-1) \beta+1] a_{n} \leq 2 \beta(1-\alpha) .
$$

Hence the result follows.

COROLLARY. If $f(z)=\frac{1}{z}+\sum_{n=1}^{\infty} a_{n} z^{n}, a_{n} \geq 0$, is in $\Sigma_{P}^{\star}(\alpha, \beta)$, then

$$
a_{n} \leq \frac{2 \beta(1-\alpha)}{(1+\beta) n+(2 \alpha-1) \beta+1}, n=1,2, \ldots
$$

with equality for each $n$, for functions of the form

$$
f_{n}(z)=\frac{1}{z}+\frac{2 \beta(1-\alpha)}{(1+\beta) n+(2 \alpha-1) \beta+1} z^{n}
$$

REMARK. The coefficient estimates for functions of the class $\sum_{P}^{*}(\alpha)$ determined in [7] follow from corollary 1 by taking $\beta=1$.

3. Distortion properties and radius of convexity estimates

Coefficient estimates obtained in the Corollary to Theorem 2 enable us to prove

THEOREM 3. If $f(z) \in \Sigma_{P}^{*}(\alpha, \beta)$, then for $0<|z|=r<1$,

$$
\frac{1}{r}-\frac{\beta(1-\alpha)}{1+\alpha \beta} r<|f(z)| \leq \frac{1}{r}+\frac{\beta(1-\alpha)}{1+\alpha \beta} r
$$

where equality holds for the function

$$
f_{1}(z)=\frac{1}{z}+\frac{\beta(1-\alpha)}{1+\alpha \beta} z \text { at } z=i r, r .
$$

Proof. Suppose $f(z)$ is in $\Sigma_{P}^{\star}(\alpha, \beta)$. In view of Theorem 2, we have (3.3)

$$
\sum_{n=1}^{\infty} a_{n} \leq \frac{\beta(1-\alpha)}{1+\alpha \beta} \text {. }
$$

Thus, for $0<|z|=\boldsymbol{r}<1$, 


$$
\begin{aligned}
|f(z)|=\left|\frac{1}{z}+\sum_{n=1}^{\infty} a_{n} z^{n}\right| & \leq\left|\frac{1}{z}\right|+\sum_{n=1}^{\infty} a_{n}|z|^{n} \leq \frac{1}{r}+r \sum_{n=1}^{\infty} a_{n} \\
& \left.\leq \frac{1}{r}+\frac{\beta(1-\alpha)}{1+\alpha \beta} r, \text { by } \quad 3.3\right) .
\end{aligned}
$$

This gives the right hand inequality of (3.1). Also

$$
\begin{aligned}
|f(z)| & \geq \frac{1}{r}-\sum_{n=1}^{\infty} a_{n} r^{n} \geq \frac{1}{r}-r \sum_{n=1}^{\infty} a_{n} \\
& \geq \frac{1}{r}-\frac{\beta(1-\alpha)}{1+\alpha \beta} r
\end{aligned}
$$

which gives the left hand side of (3.1).

It can be easily seen that the function $f_{1}(z)$ defined by (3.2) is extremal for the theorem.

THEOREM 4. If $f(z)$ is in $\Sigma_{P}^{*}(\alpha, \beta)$, then $f(z)$ is meromorphically convex of order $\delta(0 \leq \delta<1)$ in $|z|<r=r(\alpha, \beta, \delta)$, where

$$
r(\alpha, \beta, \delta)=\inf _{n}\left\{\frac{(1-\delta)((1+\beta) n+(2 \alpha-1) \beta+1)}{2 \beta(1-\alpha) n(n+2-\delta)}\right\}^{\frac{1}{n+1}}, n=1,2, \ldots
$$

The bound for $|z|$ is sharp for each $n$, with the extremal function being of the form (2.5).

Proof. Let $f(z) \in \Sigma_{\mathcal{P}}^{*}(\alpha, \beta)$. Then, by Theorem 2

$$
\sum_{n=1}^{\infty} \frac{[(1+\beta) n+(2 \alpha-1) \beta+1]}{2 \beta(1-\alpha)} a_{n} \leq 1 \text {. }
$$

In view of $(1.3)$, it is sufficient to show that

$$
\left|2+\frac{z f^{\prime \prime}(z)}{f^{\prime}(z)}\right| \leq 1-\delta \text { for }|z|<r(\alpha, \beta, \delta) \text {. }
$$

or, equivalently, to show that 


$$
\left|\frac{f^{\prime}(z)+\left(z f^{\prime}(z)\right)^{\prime}}{f^{\prime}(z)}\right| \leq 1-\delta \text { for }|z|<r(\alpha, \beta, \delta),
$$

where $r(\alpha, \beta, \delta)$ is as specified in the statement of the theorem.

Substituting the series expansions for $f^{\prime}(z)$ and $\left(z f^{\prime}(z)\right)^{\prime}$ in the left hand side of (3.6) we have

$$
\left|\frac{\sum_{n=1}^{\infty} n(n+1) a_{n} z^{n-1}}{-\frac{1}{z^{2}}+\sum_{n=1}^{\infty} n a_{n} z^{n-1}}\right| \leq \frac{\sum_{n=1}^{\infty} n(n+1) a_{n}|z|^{n+1}}{1-\sum_{n=1}^{\infty} n a_{n}|z|^{n+1}} .
$$

This will be bounded by $1-\delta$ if

$$
\sum_{n=1}^{\infty} \frac{n(n-2-\delta)}{1-\delta} a_{n}|z|^{n+1} \leq 1
$$

In view of (3.5), it follows that (3.7) is true if

$$
\frac{n(n+2-\delta)}{1-\delta}|z|^{n+1} \leq \frac{(1+\beta) n+(2 \alpha-1) \beta+1}{2 \beta(1-\alpha)}, n=1,2, \ldots .
$$

or

$$
|z| \leq\left\{\frac{(1-\delta)((1+\beta) n+(2 \alpha-1) \beta+1)}{2 \beta(1-\alpha) n(n+2-\delta)}\right\}^{\frac{1}{n+1}}, n=1,2, \ldots
$$

Setting $|z|=r(\alpha, \beta, \delta)$ in (3.8), the result follows.

Sharpness can be verified easily.

\section{Convex linear combinations}

In this section we shall prove that the class $\sum_{P}^{\star}(\alpha, \beta)$ is closed under convex linear combinations.

THEOREM 5. Let $f_{O}(z)=\frac{1}{z}$ and

$$
f_{n}(z)=\frac{1}{z}+\frac{2 \beta(1-\alpha)}{(1+\beta) n+(2 \alpha-1) \beta+1} z^{n}, n=1,2, \ldots .
$$


Then $f(z) \in \Sigma_{P}^{*}(\alpha, \beta)$ if and only if it can be expressed in the form

$$
f(z)=\sum_{n=0}^{\infty} \lambda_{n} f_{n}(z) \text { where } \lambda_{n} \geq 0 \text { and } \sum_{n=0}^{\infty} \lambda_{n}=1 \text {. }
$$

Proof. Let

$$
f(z)=\sum_{n=0}^{\infty} \lambda_{n} f_{n}(z) \text { with } \lambda_{n} \geq 0 \text { and } \sum_{n=0}^{\infty} \lambda_{n}=1 .
$$

Then

$$
\begin{aligned}
f(z) & =\sum_{n=0}^{\infty} \lambda_{n} f_{n}(z)=\lambda_{0} f_{0}(z)+\sum_{n=1}^{\infty} \lambda_{n} f_{n}(z) \\
& =\left(1-\sum_{n=1}^{\infty} \lambda_{n}\right) f_{0}(z)+\sum_{n=1}^{\infty} \lambda_{n} f_{n}(z) \\
& =\left(1-\sum_{n=1}^{\infty} \lambda_{n}\right) \frac{1}{z}+\sum_{n=1}^{\infty} \lambda_{n}\left(\frac{1}{z}+\frac{2 \beta(1-\alpha)}{(1+\beta) n+(2 \alpha-1) \beta+1} z^{n}\right) \\
& =\frac{1}{z}+\sum_{n=1}^{\infty} \lambda_{n} \frac{2 \beta(1-\alpha)}{(1+\beta) n+(2 \alpha-1) \beta+1} z^{n} .
\end{aligned}
$$

Since

$$
\sum_{n=1}^{\infty} \frac{(1+\beta) n+(2 \alpha-1) \beta+1}{2 \beta(1-\alpha)} \lambda_{n} \frac{2 \beta(1-\alpha)}{(1+\beta) n+(2 \alpha-1) \beta+1}=\sum_{n=1}^{\infty} \lambda_{n}=1-\lambda_{0} \leq 1 \text {, }
$$

by Theorem 2, $f(z) \in \Sigma_{P}^{\star}(\alpha, \beta)$.

Conversely, suppose $f(z) \in \Sigma_{P}^{\star}(\alpha, \beta)$. Since

$$
a_{n} \leq \frac{2 \beta(1-\alpha)}{(1+\beta) n+(2 \alpha-1) \beta+1}, n=1,2, \ldots
$$

setting $\quad \lambda_{n}=\frac{(1+\beta) n+(2 \alpha-1) \beta+1}{2 \beta(1-\alpha)} a_{n}, n=1,2, \ldots$, and $\lambda_{0}=1-\sum_{n=1}^{\infty} \lambda_{n}$, it follows that $f(z)=\sum_{n=0}^{\infty} \lambda_{n} f_{n}(z)$. This completes the proof of the theorem. 
THEOREM 6. The class $\Sigma_{P}^{*}(\alpha, \beta)$ is closed under convex linear combinations.

$$
\text { Proof. Suppose } f^{(i)}(z)=\frac{1}{z}+\sum_{n=1}^{\infty} a_{n}^{(i)} z^{n},(i=1,2) \text { are in }
$$
$\Sigma_{P}^{\star}(\alpha, \beta) \cdot$ Let

$$
f(z)=(1-t) f^{(1)}(z)+t f^{(2)}(z), 0 \leq t \leq 1 .
$$

Then

$$
f(z)=\frac{1}{z}+\sum_{n=1}^{\infty}\left[(1-t) a_{n}^{(1)}+t a_{n}^{(2)}\right] z^{n} .
$$

In view of Theorem 2, we have

$$
\begin{aligned}
& \sum_{n=1}^{\infty}[(1+\beta) n+(2 \alpha-1) \beta+1]\left((1-t) a_{n}^{(1)}+t a_{n}^{(2)}\right) \\
& \quad=(1-t) \sum_{n=1}^{\infty}[(1+\beta) n+(2 \alpha-1) \beta+1] a_{n}^{(1)}+t \sum_{n=1}^{\infty}[(1+\beta) n+(2 \alpha-1) \beta+1] a_{n}^{(2)} \\
& \quad \leq(1-t) 2 \beta(1-\alpha)+t 2 \beta(1-\alpha)=2 \beta(1-\alpha)
\end{aligned}
$$

which shows that $f \in \Sigma_{P}^{*}(\alpha, \beta)$. Hence the theorem.

\section{Integral transforms}

In this section we consider integral transforms of functions in $\Sigma_{P}^{\star}(\alpha, \beta)$ of the type considered by Bajpai [1] and Goel and Sohi [3].

THEOREM 7. If $f(z)$ is in $\Sigma_{P}^{*}(\alpha, \beta)$, then the integral transforms

$$
F_{c}(z)=c \int_{0}^{1} u^{c} f(u z) d u, 0<c<\infty \text {, }
$$

are in $\Sigma_{\vec{P}}^{*}(\delta)$, where

$$
\delta=\delta(\alpha, \beta, c)=\frac{(1+\alpha \beta)(c+2)-\beta c(1-\alpha)}{\beta c(1-\alpha)+(1+\alpha \beta)(c+2)} .
$$

The result is best possible for the function $f(z)=\frac{1}{z}+\frac{\beta(1-\alpha)}{1+\alpha \beta} z$. 
Proof. Suppose $f(z)=\frac{1}{z}+\sum_{n=1}^{\infty} a_{n} z^{n} \in \Sigma_{P}^{*}(\alpha, \beta)$. We have

$$
F_{c}(z)=c \int_{0}^{1} u^{c} f(u z) d u=\frac{1}{z}+\sum_{n=1}^{\infty} \frac{c a_{n}}{n+c+1} z^{n}
$$

It is sufficient to show that

$$
\sum_{n=1}^{\infty} \frac{n+\delta}{1-\delta} \frac{c a_{n}}{n+c+1} \leq 1
$$

Since $f(z) \in \Sigma_{P}^{\star}(\alpha, \beta)$, we have

$$
\sum_{n=1}^{\infty} \frac{(1+\beta) n+(2 \alpha-1) \beta+1}{2 \beta(1-\alpha)} a_{n} \leq 1 \text {. }
$$

Thus (5.3) will be satisfied if

$$
\frac{(n+\delta) c}{(1-\delta)(n+c+1)} \leq \frac{(1+\beta) n+(2 \alpha-1) \beta+1}{2 \beta(1-\alpha)} \text { for each } n
$$

or

$$
\delta \leq \frac{((1+\beta) n+(2 \alpha-1) \beta+1)(n+c+1)-2 \beta(1-\alpha) c n}{2 \beta(1-\alpha) c+((1+\beta) n+(2 \alpha-1) \beta+1)(n+c+1)} .
$$

Since the right hand side of (5.5) is an increasing function of $n$, putting $n=1$ in (5.5) we get

$$
\delta \leq \frac{(1+\alpha \beta)(2+c)-\beta(1-\alpha) c}{(1+\alpha \beta)(2+c)+\beta(1-\alpha) c} .
$$

Hence the theorem.

REMARK. It is interesting to note that for $c=1$ and $(\alpha, \beta)=(0,1)$, Theorem 7 gives that if $f \in \Sigma_{P}^{\star}(0,1)$ then $F_{1}(z)=\int_{0}^{1} u f(u z) d u$ is in $\Sigma_{\hat{P}}^{\star}\left(\frac{1}{2}\right)$. 


\section{Convolution properties}

Robertson [12] has shown that if $f(z)=\frac{1}{z}+\sum_{n=1}^{\infty} a_{n^{z^{n}}}$ and $g(z)=\frac{1}{z}+\sum_{n=1}^{\infty} b_{n} z^{n}$ are in $\Sigma_{S}$ then so is their convolution $f(z) * g(z)=\frac{1}{z}+\sum_{n=1}^{\infty} a_{n} b n^{z^{n}}$. We prove the following results for functions in $\Sigma \stackrel{*}{P}(\alpha, \beta)$

THEOREM 8. If $f(z)=\frac{1}{z}+\sum_{n=1}^{\infty} a_{n} z^{n}$ and $g(z)=\frac{1}{z}+\sum_{n=1}^{\infty} b_{n} z^{n}$ are in $\Sigma_{P}^{\star}(\alpha, \beta)$ then $f(z) * g(z)=\frac{1}{z}+\sum_{n=1}^{\infty} a_{n} b_{n} z^{n}$ is in $\Sigma_{P}^{\star}(\gamma, \beta)$ where

$$
\gamma=\frac{(1+\alpha \beta)^{2}-\beta(1-\alpha)^{2}}{(1+\alpha \beta)^{2}+\beta^{2}(1-\alpha)^{2}}
$$

The result is best possible for the functions

$$
f(z)=g(z)=\frac{1}{z}+\frac{\beta(1-\alpha)}{1+\alpha \beta} z .
$$

Proof. Suppose $f(z)$ and $g(z)$ are in $\Sigma_{P}^{\star}(\alpha, \beta)$. In view of Theorem 2, we have

$$
\sum_{n=1}^{\infty} \frac{(1+\beta) n+(2 \alpha-1) \beta+1}{2 \beta(1-\alpha)} a_{n} \leq 1
$$

and

$$
\sum_{n=1}^{\infty} \frac{(1+\beta) n+(2 \alpha-1) \beta+1}{2 \beta(1-\alpha)} b_{n} \leq 1 .
$$

Since $f(z)$ and $g(z)$ are regular in $D$, so is $f(z){ }^{\star} g(z)$. Further,

$$
\sum_{n=1}^{\infty} \frac{(1+\beta) n+\beta\left[2 \frac{(1+\alpha \beta)^{2}-\beta(1-\alpha)^{2}}{(1+\alpha \beta)^{2}+\beta^{2}(1-\alpha)^{2}}-1\right]+1}{2 \beta\left(1-\frac{(1+\alpha \beta)^{2}-\beta(1-\alpha)^{2}}{(1+\alpha \beta)^{2}+\beta^{2}(1-\alpha)^{2}}\right)} a_{n} b_{n}
$$




$$
\begin{aligned}
& \leq \sum_{n=1}^{\infty}\left[\frac{(1+\beta) n+(2 \alpha-1) \beta+1}{2 \beta(1-\alpha)}\right]^{2} a_{n} b_{n} \\
& \leq\left[\sum_{n=1}^{\infty} \frac{(1+\beta) n+(2 \alpha-1) \beta+1}{2 \beta(1-\alpha)} a_{n}\right)\left(\sum_{n=1}^{\infty} \frac{(1+\beta) n+(2 \alpha-1) \beta+1}{2 \beta(1-\alpha)} b_{n}\right) \\
& \leq 1
\end{aligned}
$$

in view of (6.2) and (6.3). Hence by Theorem $2, f(z) * g(z) \in \Sigma_{P}^{\star}(\alpha, \beta)$ where $\gamma$ is given by (6.1). This completes the proof of the theorem.

Putting $B=1$ in the above theorem, we get the following result determined in [7].

COROLLARY. If $f(z)=\frac{1}{z}+\sum_{n=1}^{\infty} a_{n^{2}}$ and $g(z)=\frac{1}{z}+\sum_{n=1}^{\infty} b_{n^{2}}$ are elements of $\Sigma_{P}^{\star}(\alpha)$, then $h(z)=f(z){ }^{*} g(z)=\frac{1}{z}+\sum_{n=1}^{\infty} a_{n} b n^{z^{n}}$ is an element of $\Sigma_{P}^{*}\left(\frac{2 \alpha}{1+\alpha^{2}}\right)$. The result is best possible.

REMARK. If $f(z)$ and $g(z)$ are in $\Sigma_{P}^{*}(0)$, then, according to the corollary, $f(z){ }^{*} g(z)$ is also in $\Sigma_{P}^{*}(0)$; the result is best possible. This is in sharp contrast with the corresponding result for starlike functions with negative coefficients (cf. Remark following Theorem 1 of Schild and Silverman [14]).

Using similar arguments as in the proof of Theorem 8, we can obtain the following result.

THEOREM 9. If $f(z) \in \Sigma_{P}^{\star}(\alpha, \beta)$ and $g(z) \in \Sigma_{P}^{\star}(\gamma, \beta)$, then $f(z) * g(z) \in \Sigma_{P}^{\star}(\tau, B)$ where

$$
\tau=\frac{(1+\alpha \beta)(1+\beta \gamma)-\beta(1-\alpha)(1-\gamma)}{(1+\alpha \beta)(1+\beta \gamma)+\beta^{2}(1-\alpha)(1-\gamma)} .
$$

The result is best possible for

$$
f(z)=\frac{1}{z}+\frac{\beta(1-\alpha)}{1+\alpha \beta} z \text { and } g(z)=\frac{1}{z}+\frac{\beta(1-\gamma)}{i+\gamma \beta} z \text {. }
$$


THEOREM 10. If $f(z)=\frac{1}{z}+\sum_{n=1}^{\infty} a_{n^{2}} z^{n} \in \Sigma_{P}^{*}(\alpha, \beta)$ and $g(z)=\frac{1}{z}+\sum_{n=1}^{\infty} b_{b} z^{n}$ with $\left|b_{n}\right| \leq 1, n=1,2, \ldots$, then $f(z){ }^{*} g(z) \in \Sigma *(\alpha, \beta)$

Proof. Since

$$
\begin{gathered}
\sum_{n=1}^{\infty} \frac{(1+\beta) n+(2 \alpha-1) \beta+1}{2 \beta(1-\alpha)}\left|a_{n} b_{n}\right|=\sum_{n=1}^{\infty} \frac{(1+\beta) n+(2 \alpha-1) \beta+1}{2 \beta(1-\alpha)} a_{n}\left|b_{n}\right| \\
\leq \sum_{n=1}^{\infty} \frac{(1+\beta) n+(2 \alpha-1) \beta+1}{2 \beta(1-\alpha)} a_{n} \leq 1
\end{gathered}
$$

by Theorem 1 it follows that $f(z) * g(z) \in \Sigma^{\star}(\alpha, \beta)$.

REMARK. $g(z)$ need not be univalent in the above theorem. For example, if $g(z)=\frac{1}{z}-\frac{a}{a+b} z^{2}$ where $0<b<a$, then $\left|\frac{-a}{a+b}\right|<1$ but $g^{\prime}(z)=-\frac{1}{z^{2}}-\frac{2 a}{a+b} z=0$ for $z=-\left(\frac{a+b}{2 a}\right)^{1 / 3}$ which lies inside $D$.

Thus $g(z)$ is not univalent in $D$.

THEOREM 11. If $f(z)$ and $g(z)$ are in $\Sigma_{P}^{*}(\alpha, \beta)$ and $1+3 \alpha \beta-2 \beta \geq 0$, then

$$
F(z)=\frac{1}{z}+\sum_{n=1}^{\infty}\left(a_{n}^{2}+b_{n}^{2}\right) z^{n}
$$

also belongs to $\Sigma_{P}^{\star}(\alpha, \beta)$.

Proof. Since $f(z) \in \Sigma_{P}^{\star}(\alpha, \beta)$ we have

$$
\sum_{n=1}^{\infty} \frac{(1+\beta) n+(2 \alpha-1) \beta+1}{2 \beta(1-\alpha)} a_{n} \leq 1 \text {. }
$$

Therefore,

$$
\sum_{n=1}^{\infty}\left(\frac{(1+\beta) n+(2 \alpha-1) \beta+1}{2 \beta(1-\alpha)}\right) a_{n}^{2} \leq 1 .
$$

Similarly 


$$
\sum_{n=1}^{\infty}\left(\frac{(1+\beta) n+(2 \alpha-1) \beta+1}{2 \beta(1-\alpha)}\right)^{2} b_{n}^{2} \leq 1 .
$$

Hence

$$
\sum_{n=1}^{\infty} \frac{1}{2}\left(\frac{(1+\beta) n+(2 \alpha-1) \beta+1}{2 \beta(1-\alpha)}\right)^{2}\left(a_{n}^{2}+b_{n}^{2}\right) \leq 1 .
$$

In view of Theorem 2 , it is sufficient to show that

$$
\sum_{n=1}^{\infty}\left(\frac{(1+\beta) n+(2 \alpha-1) \beta+1}{2 \beta(1-\alpha)}\right)\left(a_{n}^{2}+b_{n}^{2}\right) \leq 1 .
$$

Thus (6.4) will be satisfied if, for $n=1,2, \ldots$,

$$
\frac{(1+\beta) n+(2 \alpha-1) \beta+1}{2 \beta(1-\alpha)} \leq \frac{1}{2}\left(\frac{(1+\beta) n+(2 \alpha-1) \beta+1}{2 \beta(1-\alpha)}\right)^{2}
$$

or if

$$
(1+\beta) n+6 \alpha \beta-5 \beta+1 \geq 0 \text { for } n=1,2, \ldots \text {. }
$$

The left hand side of (6.5) is an increasing function of $n$, hence (6.5) is satisfied for all $n$ if $1+3 \alpha \beta-2 \beta \geq 0$ which is true by our assumption. Hence the result.

\section{References}

[1] S.K. Bajpai, "A note on a class of meromorphic univalent functions", Rev. Roumanie Math. Pures Appl. 22 (1977), 295-297.

[2] J. Clunie, "On mexomorphic schlicht functions", J. London Math. Soc. 34 (1959), 215-216.

[3] R.M. Goel and N.S. Sohi, "On a class of meromorphic functions", Glasnik Matematicki 17 (1981), 19-28.

[4] V.P. Gupta and P.K. Jain, "Certain classes of univalent functions with negative coefficients", Bull. Austral. Math. Soc. 14 (1976), 409-416.

[5] O.P. Juneja and M.L. Mogra, "On starlike functions of order $\alpha$ and type $B$ ", Notices Amer. Math Soc. 22 (1975), A-384; Abstract No. 75T-B80. 
[6] O.R. Juneja and M.L. Mogra, "On starlike functions of order $\alpha$ and type $\beta$ ", Rev. Roumaine Math. Pures App 2.23 (1978), 751-765.

[1] O.P. Juneja and T.R. Reddy, "Meromorphic starlike univalent functions with positive coefficients" (communicated).

[8] J.E. Miller, "Convex meromorphic mappings and related functions", Proc. Amer. Math. Soc. 25 (1970), 220-228.

[9] M.I. Mogra and O.P. Juneja, "Coefficient estimates for starlike functions", Bulz. Austral. Math. Soc. 16 (1977), 415-425.

[10] K.S. Padmanabhan, "On certain classes of starlike functions in the unit disc", J. Indion Math. Soc. (N.S) 32 (1968), 89-103.

[11] Ch. Pommerenke, "On meromorphic starlike functions", Pacific $J$. Math. 13 (1963), 221-235.

[12] M.S. Robertson, "Convolutions of schlicht functions", Proc. Amer. Math. Soc. 13 (1962), 585-589.

[13] W.C. Royster, "Meromorphic starlike multivalent functions", Trans. Amer. Math. Soc. 107 (1963), 300-308.

[14] A. Schild and H. Silverman, "Convolution of univalent functions with negative coefficients", Ann. Univ. Mariae. Curie. SkLodwska Sect. A29 (1975), 99-107.

[15] H. Silverman, "Univalent functions with negative coefficients", Proc. Amer. Math. Soc. 51 (1975), 109-116.

[16] H. Silverman, "Extreme points of univalent functions with two fixed points", Trans. Amer. Math. Soc. 219 (1976), 387-395.

[17] H. Silverman and E.M. Silvia, "Prestarlike functions with negative coefficients", Internat. J. Math. and Math. Sci. 2 (1979), 427-439.

School of Mathematical Sciences, University of Khartoum, Khartoum, SUDAN.

Department of Mathematics, Indian Institute of Technology, Kanpur-208016, INDIA.
Department of Mathematics, Kakatiya University, Warangal-506009, AP, INDIA. 